\title{
HIGH-SPEED DATA ACQUISITION DESIGN AND IMPLEMENTION THAT IS BASED ON S7-300PLC AND WINCC
}

\author{
FangWang ${ }^{1}$, Juyang Lei ${ }^{2}$ \\ ${ }^{I}$ College of Mechanical Engineering, Shanghai University of Engineering Science, Shanghai, China \\ ${ }^{2}$ College of Mechanical Engineering, Shanghai University of Engineering Science, Shanghai, China
}

\begin{abstract}
Information technology has become a consensus today, at the same time, we need to integrate and deal with the huge data. Especially in some specific areas, such as rapid reaction system, the whole reaction process is less than 5 s. To analyze the entire reaction process to understand the parameters of the change, even with the 500ms sampling cycle, the data collection is still too little. In this case, resource consumption on the machine for communication with the controller system has been very high, coupled with data processing, images, database tasks and etc. When the scale of variable size is not much, the host computer load has been overwhelmed. The use of acquisition board to achieve high-speed data acquisition is limited by the size of the entire system limitations, and also because of the number of computer expansion slot restrictions, the final number of points are dozens. Based on the above problems we combine s7-300PLC with WinCC for high-speed collection of information to achieve the value of large data extraction.
\end{abstract}

Keywords: Information Technology, S7-300PLC, WinCC, High-speed Collection $* * *$

\section{INTRODUCTION OF S7-300PLC}

\subsection{Configuration Design of S7-300PLC}

The development of PLC is very fast, because of its high reliability, small size and application simpler, it is a particularly suitable for industrial environments, engineering and technical personnel and general-oriented industrial computer workers [1]. PLC is not only a single system, but is often with other technologies in the actual production and work. For example, computer technology, communication connection technology and so on. Its advanced real-time monitoring and control functions, combined with the diversification of other technologies, make it possible to harness almost all of the industrial automation production.

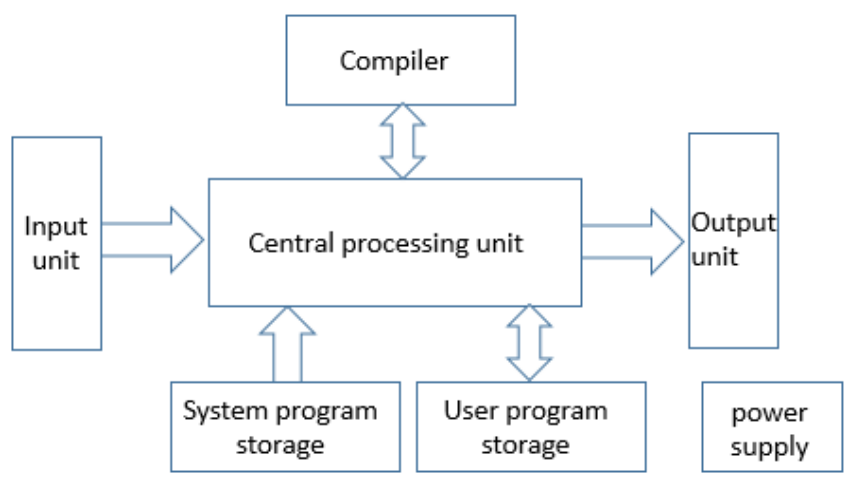

Fig-1: S7-300 system

First of all, that is to establish an analog PLC hardware system in the STEP7 configuration [2], all modules in RANK are in a virtual form of the establishment, through the input power supply, CPU input module and output module is to PLC specific configuration, and in the configuration process, the specific parameters will be stored in the data block SDB.

\subsection{CPU Module Parameters Setting}

CPU module is the core of the control system, and is responsible for the central control system, storage and executable programs. The CPU has four operating modes: stop, start, run and hold. The various parameters of the module in the CPU can be detailed in STEP7. In the SIMATIC Manager [3], click on the hardware button, there will be configuration screen, select the line where the property CPU debugging, set the required parameters and requirements. Here is the choice of CPU 413-2DP type.

\subsection{Input and Output of Analog Modules}

In the 5 and 6 slot of rank, I add input and output modules, the parameter settings must be set when the CUP state is stop. If the CUP is in the run mode, parameters will be transferred to each module. Just like the CUP settings, enter the hardware system in the SIMATIC Manager, doubleclick the input and output modules in the card slot, and click the Address tab to set the start byte address and input and output parameters. The DI and DO modules are laid in 7 and 8 respectively. The specific parameters are as follows: 


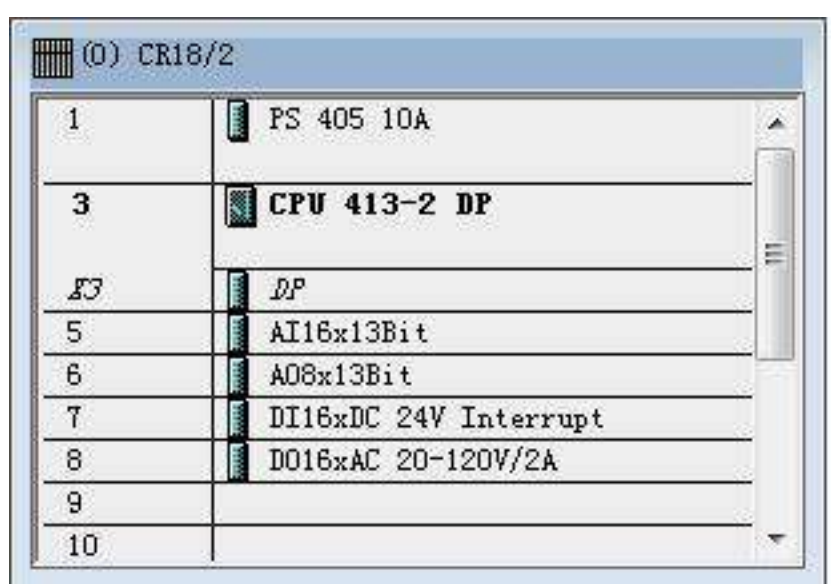

Fig-2: specific parameters

\section{DESIGN AND IMPLEMENTATION OF S7-300} WINCC HIGH SPEED DATA ACQUISITION

\section{PROGRAM}

In this paper, through the establishment of the original data type, high-speed data acquisition system is established. To achieve data acquisition process the use of "double buffer" mode, the main program interrupt mode call OB35 module to achieve continuous centralized control, and real-time monitoring through WINCC [4] simulation sine function graphics are needed.

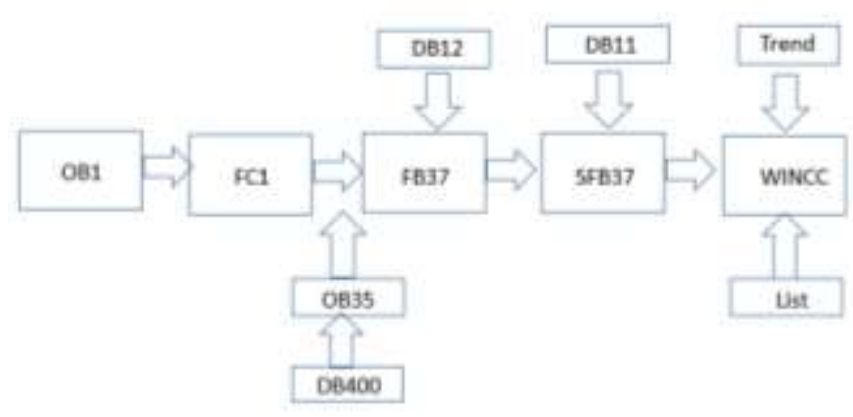

Fig-3: High-speed data acquisition flow chart

\subsection{Design of S7-300 Software Programming and} Function Module

S7-300 software programming is in the STEP7, the use of ladder, or other table or flow chart programming for the PLC through OB1 module to its operating system and user programs can be connected to form a "linear Design ".This method can organize all the data blocks of OB1 [5] in an orderly manner. PLC can regularly call this one. FB, DB and other functional blocks can be organized OB1 call and the program has been structured.

The specific operation is as follows: First, the establishment of OB1 module, is deemed as the main program module, followed by CPU start. In addition to OB90, OB1 has the lowest priority and can be interrupted by other OB modules.

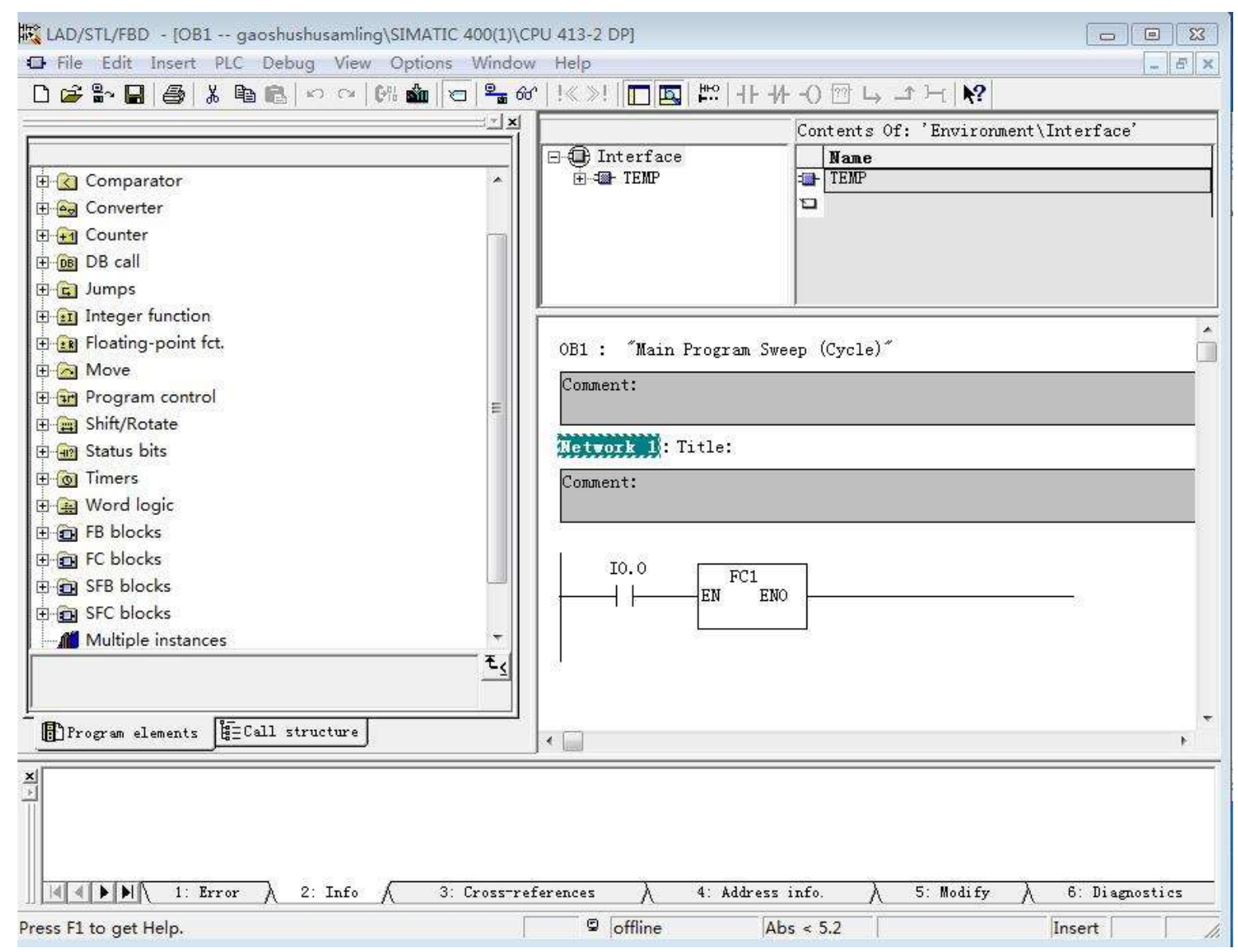

Fig-4: OB1 data block components 
FC is as a shared data block, although it can be called, but it is temporary, similar to a function, what is more, FC1 is attached to the FB37, and the FB37 data sources is from DB11.

\section{WINCC CONFIGURATION}

WinCC (Windows Control Center) is based on the worldwide use of system design, and can communicate with third-party controller. As a common application, suitable for all industrial solutions, also can be integrated into all automation solutions for the industrial configuration and research. Under normal circumstances, WinCC can be used in conjunction with the PLC contact, you can make PLC create a variety of data blocks to establish contact with them. First of all, I establish an original data type by using a sinusoidal function of the image, moreover the sin function will be changed to achieve the subject of data collection, while PLC reads the conversion of the DLL [6]constantly. The address of PLC and WinCCare the same, the AR_ID and AR_ID_subnumber function are established in PLC. AR_ID is as a fixed value to be marked, and for the AR_ID_subnumber there will be more than one variable and parameter settings. The connection will be successful, then the PLC will be required to send the program to the WinCC database to perform further operations.

According to the requirements of the establishment of the archive variable, first I choose the same time interval with the process of marking the value of the type, which is characterized by saving storage space and simplified communication.

\subsection{Create a Data Block}

The establishment of the data block DB10, its main role is for the step and WinCC of the background data block, and make the appropriate call. In the table, Headtype 1 Word has the first value of 0 , the data length 1-2000, SinData1 has 22 data, and REAL is 4 bytes. Therefore, the header DB10 has
$4 * 2000+22=8022$ data. DB10 and DB400 are used as shared data, the intermediate variables of DB is for storing user data and procedures for global variables. The DB maximum capacity, S7-300, 32KB, S7-400 of the $400 \mathrm{~KB}$. The valid data block is stored in the background, but for the instance data block, it is just a module. The instance DB FB, SFB[7] and global variables are assigned.

MW14 = 8022 that represents a total of 8022 bytes of data. In addition to holding 22 message head, we create 200 REAL-type data, the length of which is 8000 bytes.

\subsection{WinCC Accepts the Processing}

As the WinCC accepts the processing, the main is the integration of the database and processing, which is a linked process. Through the background of the archive data to connect by using the function as a receiver [8], and use DLL to extract and deal. And finally record the process to achieve the acceptance of WinCC.

The data packets sent by the S7 after the removal of the WinCC project are stored in the back-end database. Then start logging the tags in the WinCC Explorer to the operating system so that the call is controlled in the WinCC Graphic Editor Online Trend Online Data Curve, also the value of the variable file during the selection process, when running the graphic you can see the high speed data acquisition. Data is sent from the buffer to WinCC. First of all, 2000 bytes of data by specifying the length of 2000 bytes of data are inserted in 2000, by using FC call in OB1 in the operation of the program to reach the connection. When finished, reset the half-full flag.

According to previous research conclusions, the Block to download, and then open OB1 into the emulator, it is time to run. WinCC is into the graphical editor, finally there sinusoidal suggests the acquisition is successful.

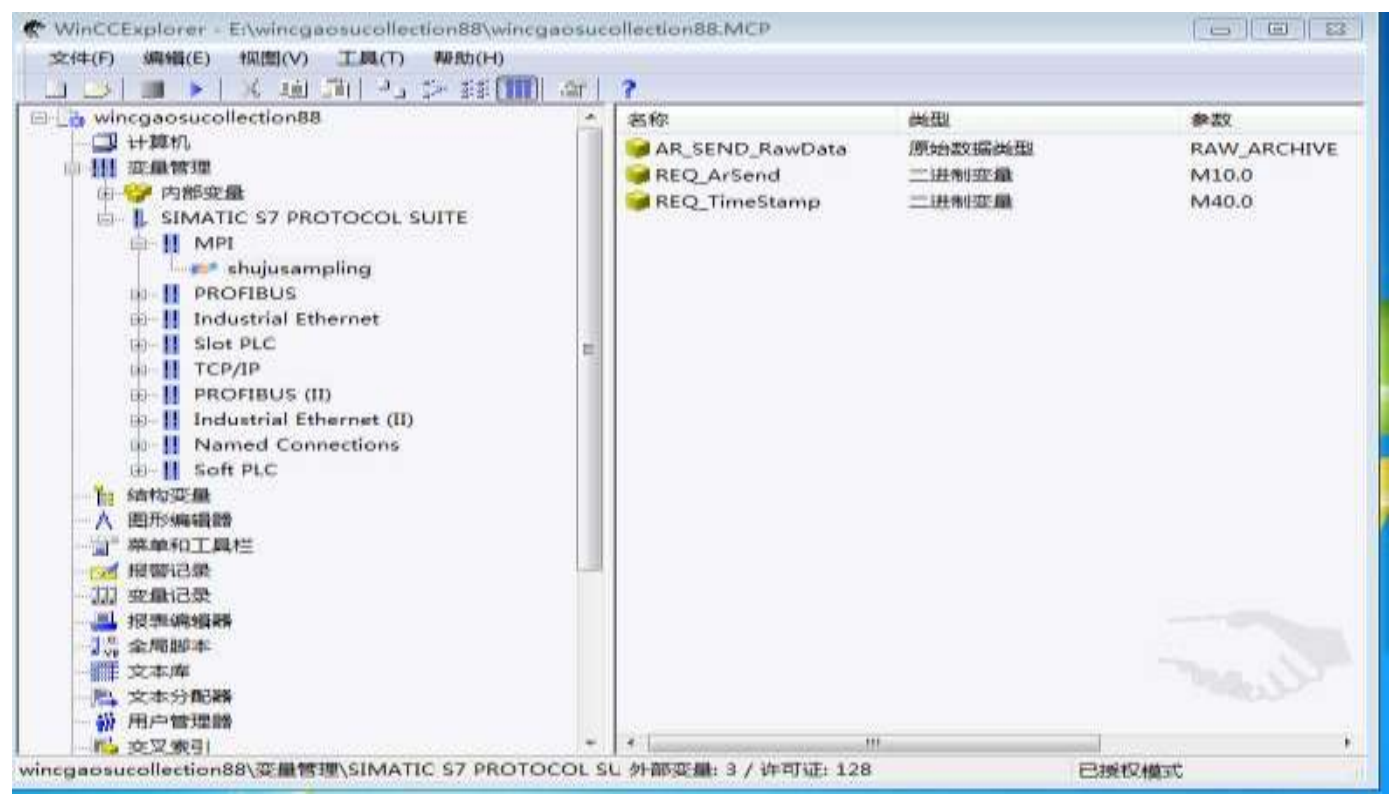

Fig-5: WinCC internal configuration 


\section{RESULTS}

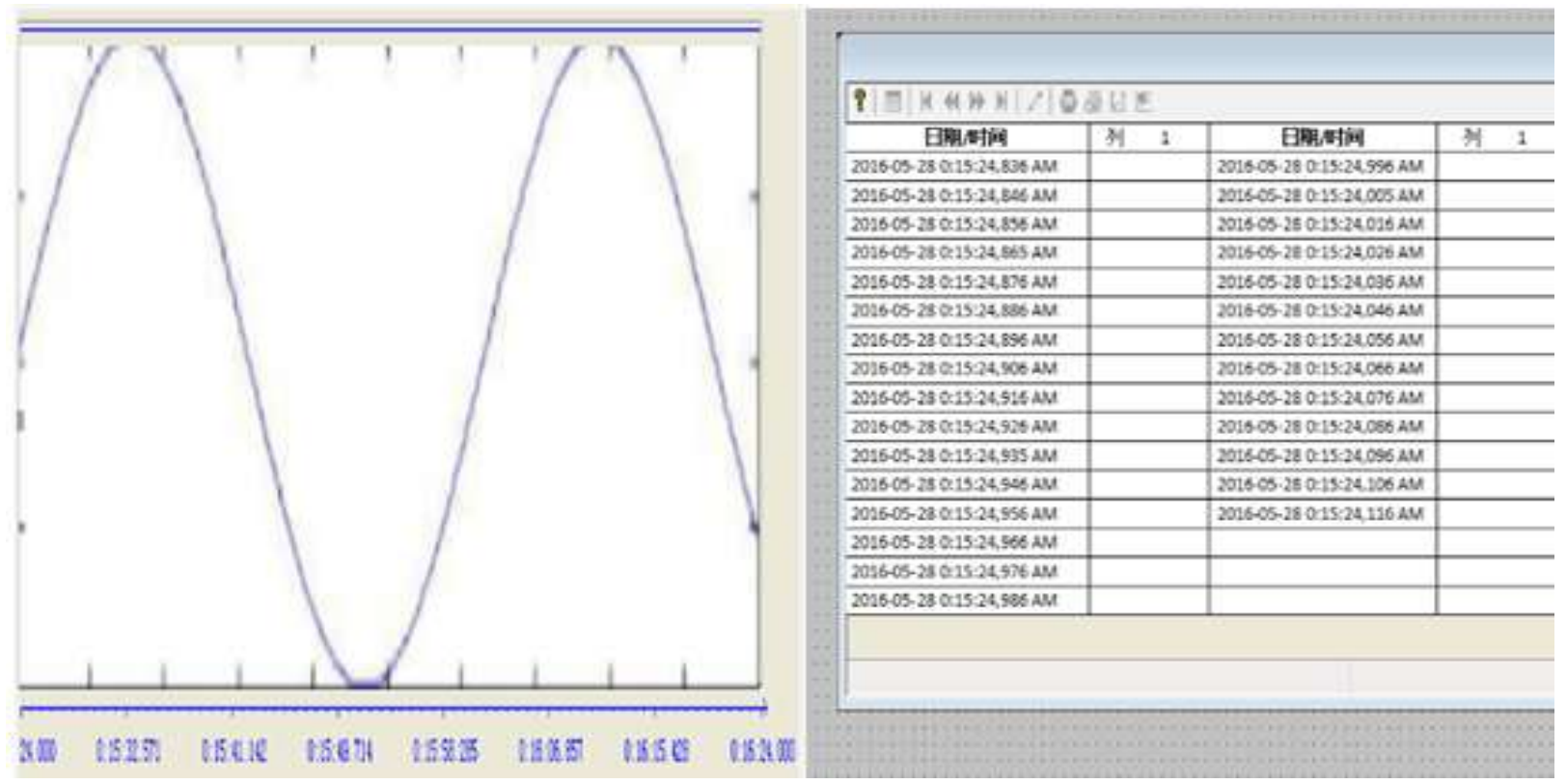

Fig-6: Acquisition effect map

As shown in Figure 12.2, the data acquisition is realized and the cycle frequency is maintained at $10 \mathrm{~ms}$, which meets the standard of high-speed acquisition.

\section{CONCLUSION}

This design uses the S7-300 and WinCC two languages, the first use of the data acquisition plc fast, followed by the use of the buffer zone settings to better deal with large data, compared with the conventional approach greatly reflects its superior performance, For the future design and production played a decisive role. The design of the title is based on WinCC configuration software design. Although the host computer design, complete the provisions of the starting and stopping and self-protection and related content and manual control to the host computer to complete. If only from the host computer interface design, its content and difficulty is very limited, but the so-called control can not only rely on the host computer to complete, but also the next bit machine PLC and belt conveyor with the completion of the common.

\section{REFERENCES}

[1]. District Yi-shan. Computer Network Engineering Manual. Beijing: Publishing House of Electronics Industry, 1993.

[2]. Yang Luming, Lei Yajun. Application of Configuration Software WinCC in Automatic Monitoring System. Computing Technology and Automation, 2003,22 (4)

[3]. SIMATIC System Software for S7-300 / 400 Systemand Standard Functions,378-379.

[4]. WinCC Manual (Volume 1): 284- 287.

[5]. Bian Chunyuan, Ren Shuangyan, Man Yongkui, et al. Development Guideline of S7-300 / 400PLC [M] .Beijing: Mechanical Industry Press, 2007.472-473.

[6]. Li Wenlei, Liu Shirong, Chen Zheng, etc.MATLAB in the application of automatic control theory experiment
.Experimental Technology and Management, 2006, 23 (2): 68-71.

[7]. Wang Shuguang, Yang Chunjie, Wei Qiuyue, etc. S7300 / 400PLC entry and development examples. Beijing: People's Posts and Telecommunications Press, 2009,2,9495.

[8]. Siemens (China) Co., Ltd. Automation and Drives Group. Easy to understand Siemens WinCC V6. Beijing: Aerospace University Press, 2004.35-40. 